\title{
Effect of Vision Therapy on Measures of Oculomotor Function in Patients Presenting with Post-Concussion Syndrome
}

\author{
Paul Rollett, OD, FCOVD \\ Okanagan Vision Therapy \\ Optometry
}

Garrett Morandi, PhD

University of Saskatchewan

\begin{abstract}
Oculomotor dysfunction is a common symptom of post-concussion syndrome (PCS). In this study, the efficacy of Vision Therapy (VT) for the treatment of PCS-related symptoms of the visual system was investigated retrospectively. Overall, 56 patients were selected, all of whom presented with clinical impairment of at least one measure of oculomotor functioning. Activities related to VT were wide-ranging and case-dependent, but all aimed to improve one of the five main areas of visual function. Following the completion of VT, all patients demonstrated statistically or clinically significant improvements, as defined by the use of Morgan's norms, in at least one measure of oculomotor functioning related to PCS. In general, improvements in measures of oculomotor functioning were greatest for near point of convergence, vergence facility and accommodative facility. Patients who received 20 sessions of VT had improved and less variable outcomes than those who received 5-10 sessions of VT. In addition, VT improved symptoms of visual discomfort in patients presenting with PCS. The results of this retrospective analysis demonstrate significant improvements in measured outcomes for all patients who received VT, and support VT as a treatment option for symptoms of PCS.
\end{abstract}

\section{INTRODUCTION}

Mild traumatic brain injury (TBI), such as that sustained due to a blow to the head, violent shaking or movement of the head or body, can lead to symptoms of concussion. ${ }^{1}$ Following injury, symptoms of a concussion generally manifest in the first 7-10 days and can persist for up to one year or more. Symptoms of a concussion are referred to collectively as post-concussion syndrome (PCS). ${ }^{1}$ Symptoms of PCS include, but are not limited to, headaches, dizziness, fatigue, noise and light sensitivity, and issues associated with the visual system. In particular, between $11 \%$ and $54 \%$ of patients in cohort studies of soldiers or patients of auto accidents presenting with symptoms of TBI have been diagnosed with one or more oculomotor dysfunctions including convergence insufficiency, accommodative insufficiency, impaired version movement, and minor ocular alignment issues. ${ }^{2-10}$ In addition, $46 \%$ of adolescents presenting with symptoms of a concussion had more than one vision-related diagnosis such as accommodative disorder, convergence insufficiency or saccadic dysfunction. ${ }^{11}$ Convergence insufficiency is a prevalent vision disorder characterized by reduced fusional convergence amplitude and receded near point of convergence, which can manifest as blurred vision, eye strain and headaches when engaging in near work. ${ }^{12-14}$ Previous work has suggested that symptoms of convergence insufficiency and related oculomotor issues are likely due to excessive convergence compensation for large exophoric postures in near vision. ${ }^{15}$

Optometric vision therapy (VT) has emerged as a promising treatment option for patients presenting with symptoms of PCS or TBI.,5 For example, in a retrospective study, $90 \%$ of participants receiving VT for an acquired brain injury demonstrated complete or significant improvements in at least one of the recorded symptoms of acquired brain injury, and these changes 
persisted when measured 2 months or more following completion of VT. ${ }^{16}$ In addition, similar improvements in measures of oculomotor functioning have been observed in most patients receiving VT for PCS or TBI.,5,17-20 Furthermore, VT has been demonstrated to improve measures of concentration, memory and comprehension in patients presenting with symptoms of PCS or TBI. ${ }^{20}$ By use of the convergence insufficiency treatment trial symptom survey (CISS), Alvarez et al. ${ }^{21}$ demonstrated significant improvements in patients presenting with convergence insufficiency following $18 \mathrm{~h}$ of vergence training, and their scores were similar to those in control patients. In addition, the work by Alvarez et al..$^{21}$ further supports the notion that convergence insufficiency is due to significantly reduced convergence peak velocity, and vergence training leads to increased functional activity. Furthermore, Scheiman and Wick..$^{15}$ demonstrated that office-based vergence and accommodative therapy with home practice can result in sustained improvements in symptoms of convergence insufficiency.

Although there exists evidence to support VT in the treatment of PCS- or TBI-related symptoms, additional data to support a positive role for VT in the treatment of traumatic brain injury would be timely.,22 Therefore, in this work, we present the results of a retrospective analysis of the efficacy of VT treatment for improving various oculomotor functions in patients presenting with symptoms of PCS.

METHODS

Patients provided their express consent before treatment for oculomotor dysfunction was initiated.

\section{PATIENT EVALUATION}

Patients diagnosed with PCS by a physician were given a thorough oculo-visual assessment. Vision testing relevant to this study included assessments of near point of convergence (NPC), vergence facility (12 Base Out and 3 Base In), accommodative facility by use of $+/-2.00 D$ lens power (age-dependent), stereopsis measures at near (40cm) and distance (6 metres) by use of Randot Stereopsis testing, King-Devick Saccadic Testing, positive fusional vergence ranges at near $(40 \mathrm{~cm})$ and distance (6 metres), negative fusional vergence ranges at near $(40 \mathrm{~cm})$ and distance $(6$ metres) and completion of CISS before and one week following treatment. All tests were performed as described by Elliott. ${ }^{23}$ Patients presenting with nerve palsies, extraocular muscle restrictions, elevated intraocular pressure or gross visual field loss as determined by confrontation were excluded from the study.

\section{VISION THERAPY TREATMENT REGIMEN}

Following assessment, the number of sessions required was selected on a per-patient basis based on best judgement, previous literature and occasionally by approval from $3^{\text {rd }}$-party payors. ${ }^{2,15,24}$ Vision therapy consisted of weekly onehour sessions at the clinic performed by trained vision therapists and prescribed daily sessions of 15 minutes at home. Activities prescribed were wide-ranging and case-dependent, but all aimed to improve one of five main areas of visual function: central-peripheral visual integration (spatial awareness), eye teaming (converging and diverging), eye focusing (accommodation), eye tracking (smooth pursuits and saccades), and motion processing (tracking of moving objects within a busy visual scene). The details of the procedures used throughout therapy have been described previously. ${ }^{15,25}$

\section{STATISTICAL ANALYSIS}

Patient data were collected between January 1, 2017, and December 31, 2017, from all patients receiving vision therapy for PCS $(n=56)$. Individual scores of the 56 patients were averaged across all measured outcomes and values before (pre-) and after (post-) VT intervention were compared. Patient outcomes pre- and post-VT were compared by use of the dependent t-test $(p<0.05)$. Endpoints that did not meet the assumptions of normality or equal variance were compared by use of the Wilcoxon signed rank test as suggested by Rosner et al. ${ }^{26}$ To assess the clinical significance of patient outcomes, measured or recorded values were compared with the relevant literature, namely Morgan's table of norms. ${ }^{15}$

\section{RESULTS}

\section{Data overview}

Overall, 56 patients were included in the retrospective analysis and descriptive statistics are presented in Table 1. Patients experienced a range of events resulting in the presence of PCS, including motor vehicle accidents in 38 , sport-related injuries in 10 and falls in 8. Patient age ranged between 12 and 62 years (mean \pm SD; 42.6 \pm 13.8 ) and patients received between 5 and 20 sessions of VT $(13.8 \pm 4.70)$ to treat symptoms of PCS. The average patient program duration (15.2 weeks) was greater than the number of sessions of VT (13.8 sessions) and reflects the impact of patient appointment cancellation or delays in treatment from week-to-week on program duration. In addition, patients generally began VT treatment one month following assessment. 


\section{Measured patient outcomes}

In general, treatment of symptoms of PCS with VT resulted in significant improvements of all measured oculomotor functions (Table 2). All patients included in this study demonstrated improvement in at least one measure of oculomotor functioning. Forty-six patients presented with clinically impaired NPC and, following VT, 86\% of patients demonstrated clinically relevant improvements (Table 2). Fifty-five patients presented with clinically impaired vergence facility (Table 2) and, following VT, all patients demonstrated improved vergence facility; this improvement was clinically relevant in 36 (65\%). When assessed for accommodative facility, 20 of 22 patients presented clinically relevant impairment and, following treatment, $90 \%$ of patients demonstrated clinically significant improvements. When measured by use of the King-Devick Test, 43 patients presented clinically impaired saccadic movement and, following treatment, 26 patients (60\%) demonstrated clinically significant improvements (Table 2 ).

Forty-six patients presented with clinically relevant impairment of stereopsis when tested at distance. All patients but 3 experienced average score improvements of 50\%, and these improvements were clinically relevant in 23 (50\%). When stereopsis was assessed at near distances, 40 patients demonstrated clinically relevant impairment. Following treatment, average improvements of $45 \%$ were observed, and 30 patients (75\%) had clinically significant outcomes. Fifty-three patients had CISS symptom survey scores greater than 22. Following treatment, all patients showed average score improvements of $48 \%$, and these improvements were clinically significant in 35 (66\%) (Table 2).

\section{Patient convergence and divergence test scores}

Both positive and negative fusional vergence ranges (PFV/NFV) were assessed at distance (6 metres) and near $(40 \mathrm{~cm})$. When PFV at distance was measured, 51 patients presented with clinically impaired convergence break. Forty-nine patients had an average improvement of 11D in positive fusional break scores, and these improvements were clinically relevant in 26 (53\%). When assessed for PFV recovery at distance, 46 patients presented with clinically impaired scores. Following treatment, all but one patient had improved scores, and these improvements were clinically significant in 32 (69\%) (Table 2). When NFV at distance was assessed, 52 patients presented with clinically relevant impairment when assessed for divergence break. Following treatment, 47 patients had an observed average score improvement of $4.7 \mathrm{D}$, and this improvement was clinically significant in 35 (67\%). When assessed for NFV recovery at distance, 42 patients presented with clinically relevant impairment. All but one patient demonstrated improvements in score following VT, and these improvements were clinically significant in 40 (95\%) (Table 2).

Improvements similar to those at distance were observed for fusional vergence ranges at near. Fifty patients presented with clinically impaired PFV break. Forty-nine patients had average improved scores of 19.6 D, and these improvements were clinically relevant in 46 (94\%). When assessed for PFV recovery at near, 47 patients presented with clinically relevant impairment. Overall, patients saw an average improvement of $18.5 \mathrm{D}$, and these improvements were clinically relevant in 42 (89\%) (Table 2). When assessed for NFV ranges at near, 55 patients presented with clinically relevant deficits. Fifty-three patients saw an average improvement of $10.4 \mathrm{D}$, and these improvements were clinically significant in 30 (55\%). When assessed for NFV recovery at near, 45 patients presented with clinically relevant impairment. All but one patient demonstrated an average improvement of 12 D following VT, and these improvements were considered clinically significant in 41 (91\%) (Table 2).

\section{Effect of treatment duration}

Because patients received between 5 and 20 VT sessions, we wished to compare measures of oculomotor functioning among patient cohorts receiving 5-10, 12-17 or 20 sessions of VT (Table 3). In general, improvements in measures of oculomotor functioning were observed regardless of the duration of treatment. However, patients who received 20 sessions demonstrated greater improvements in scores than patients who received 10 or less sessions of VT. Improvements in vergence facility, stereopsis at distance, and measures of PFV and NFV at both distance and near showed the greatest improvement with a longer treatment duration (Table 3). In addition, measured outcome improvements were compared among age groups, and no trend was observed.

\section{DISCUSSION}

The effectiveness of VT in the treatment of PCS-related symptoms of the visual system was investigated retrospectively. Overall, 56 patients were included in this study, all of whom presented with clinical impairment of at least one measure of oculomotor functioning (Table 1; Table 2). Following VT, all patients demonstrated statistically or clinically significant improvements in at least one measure of oculomotor functioning related to PCS (Table 2). In general, improvements in measures of oculomotor functioning were greatest for NPC, vergence facility and accom- 
modative facility (Table 2). These results were similar to those of Ciuffreda et al., ${ }^{16}$ who reported that, in multiple studies, $87-100 \%$ of patients with an acquired brain injury saw improvements in accommodative dysfunction following VT. More recently, Thiagarajan and Ciuffreda ${ }^{18}$ demonstrated the efficacy of VT for improving amplitudes of vergence and accommodation, accuracy of saccadic eye movements, and overall reading in patients with mild TBI. ${ }^{18}$ Furthermore, improvements in NPC, PFV at near and distance, and CISS symptom scores were similar to those observed in children and a cohort with an age range similar to that in this study. ${ }^{22,24}$

Interestingly, patients who received 20 sessions of VT had improved and less-variable outcomes than those who received 5-10 sessions of VT (Table 3). These results are similar to those of the Convergence Insufficiency Treatment Trial Study Group, ${ }^{24}$ which observed that 12 weeks of VT sessions was insufficient to normalize measures of oculomotor functioning in children with mild TBI. However, it is important to note that, regardless of the program duration, measures of oculomotor functioning, such as convergence and accommodation, can be improved within as little as two to six weeks of VT. ${ }^{4,27}$

Vision therapy was found to improve symptoms of visual discomfort in patients presenting with PCS, as demonstrated by average improvements of $50 \%$ in CISS scores, similar to previous results reported by Scheiman and Wick $^{15}$ (Table 2). However, although improvements in oculomotor functioning were apparent, no outcomes related to return-to-work or activities of daily living were recorded in this study, and this limits the interpretation of the results. Caution should be exercised in interpretation of these results as there is a tendency for measured oculomotor outcomes, especially near point of convergence, to improve over time in placebo groups. ${ }^{27}$ In addition, the effects of motivation and encouragement on patient outcomes cannot be discounted. ${ }^{27}$

\section{CONCLUSION}

The results of this retrospective analysis support the findings of previous studies and demonstrate statistically and clinically significant improvements of measured oculomotor functioning in patients presenting with oculomotor deficiencies associated with PCS and support VT as a viable treatment option for associated symptoms. Furthermore, the present results suggest that longer treatment times (20 VT sessions) might improve measured outcomes compared to those in patients who receive 10 or less VT sessions; however, the impact of delayed VT treatment remains unclear. Although no placebo group was available in this study, the results demonstrate significant improvements in measured outcomes for all patients receiving VT and support VT as a treatment option for PCS. $\bullet$

\section{ACKNOWLEDGEMENTS}

The authors wish to thank the entire team at Okanagan Vision Therapy Optometry for their commitment towards providing high quality vision therapy and rehabilitation within our community.

We also wish to thank Vision Therapy Canada, COVD and OEPF for their tireless efforts working to advance the quality of vision therapy provided within clinics across the world.

DISCLAIMERS

The views expressed in the submitted article are our own and not an official position of an institution or funder.

FINANCIAL DISCLOSURES

This study was self-funded using clinical data and resources from our clinic - Okanagan Vision Therapy, Kelowna BC, Canada.

CONFLICTS OF INTEREST

The authors have no conflicts of interest to declare.

STATEMENT OF PUBLICATION

This article has not been submitted/published elsewhere.

CORRESPONDING AUTHOR

Paul Rollett, OD, FCOVD, Okanagan Vision Therapy Optometry

email: drrollett@okanaganvisiontherapy.ca 
Table 1: Summary statistics of treatment regimen and descriptive statistics of patients receiving vision therapy treatments for post-concussion syndrome $(n=56)$.

\begin{tabular}{|l|c|c|c|}
\hline Measure & Average & Standard Deviation & Range \\
\hline Age & 42.6 & 13.8 & $12-62$ \\
\hline Months since injury & 15.1 & 14.2 & $2-60$ \\
\hline $\begin{array}{l}\text { Delay in starting VT (months) } \\
\text { following assessment }\end{array}$ & 1.5 & 2 & $0-12$ \\
\hline Sessions of VT & 13.8 & 4.7 & $5-20$ \\
\hline Duration of Program (weeks) & 15.2 & 4.9 & $6-24$ \\
\hline
\end{tabular}

Table 2: Summary statistics of optometric data following vision therapy treatment for post-concussion syndrome ( $n=56$ ).

\begin{tabular}{|c|c|c|c|c|c|c|c|}
\hline \multirow[t]{2}{*}{ Measure } & \multicolumn{2}{|c|}{ Pre VT } & \multicolumn{2}{|c|}{ Post VT } & \multicolumn{3}{|c|}{$\begin{array}{c}\text { Score } \\
\text { Improvement }\end{array}$} \\
\hline & avg & std & avg & std & avg & std & range \\
\hline $\mathrm{NPC}(\mathrm{cm})$ & 13.0 & 9.3 & $3.57^{*}$ & 1.94 & $65 \%$ & 2 & $6.2-97 \%$ \\
\hline Vergence Facility (cpm) & 2.38 & 3.92 & $13.1^{*}$ & 3.18 & $83 \%$ & 27 & $0-100 \%$ \\
\hline Accomodative Facility - Binocular (cpm) $\delta$ & 3.14 & 3.06 & $10.6^{*}$ & 1.87 & $71 \%$ & 26 & $17-100 \%$ \\
\hline King Devick (s) & 76.4 & 41.0 & $51.4^{*}$ & 18.3 & $27 \%$ & 16 & $0-73 \%$ \\
\hline Stereopsis- $40 \mathrm{~cm}$ (secs of arc) & 79.8 & 40.6 & $42.6^{*}$ & 14.3 & $39 \%$ & 23 & $0-85 \%$ \\
\hline Stereopsis- 6 metres (secs of arc) & 134 & 125 & $48.2^{*}$ & 12.8 & $41 \%$ & 31 & $0-90 \%$ \\
\hline CISS (Score) & 38.2 & 11.42 & $20.0^{*}$ & 8.00 & $49 \%$ & 14 & $23-100 \%$ \\
\hline \multirow[t]{2}{*}{ Measure } & \multicolumn{2}{|c|}{ Pre VT } & \multicolumn{2}{|c|}{ Post VT } & \multicolumn{3}{|c|}{ Score Improvement } \\
\hline & avg & std & avg & std & avg & std & range \\
\hline PFV Break -D (6m) & 8.05 & 5.94 & $19.4^{*}$ & 7.43 & 11.4 & 7.4 & $0-30$ \\
\hline PFV Recovery -D (6m) & 4.36 & 4.86 & $14.8^{*}$ & 6.67 & 10.4 & 6.4 & $0-28$ \\
\hline NFV Break -D (6m) & 3.77 & 2.36 & $8.46^{*}$ & 2.98 & 4.7 & 3.3 & $0-16$ \\
\hline NFV Recovery -D (6m) & 1.09 & 2.51 & $5.96^{*}$ & 2.27 & 4.9 & 3 & $0-15$ \\
\hline PFV Break -D (40cm) & 11.4 & 7.25 & $29.6^{*}$ & 6.98 & 18.2 & 8.9 & $0-38$ \\
\hline PFV Recovery -D (40cm) & 4.46 & 6.16 & $21.3^{*}$ & 7.49 & 16.9 & 9.1 & $0-38$ \\
\hline NFV Break -D (40cm) & 11.1 & 5.99 & $21.4^{*}$ & 3.30 & 10.3 & 6.3 & $0-27$ \\
\hline NFV Recovery D (40cm) & 6.48 & 5.37 & $16.8^{*}$ & 3.45 & 10.3 & 6.2 & $-4-22$ \\
\hline
\end{tabular}

*Significant improvement in score $(\mathrm{p}<0.001)$.

$\delta n=22$, average age of $28.4 \pm 10.1$ 
Table 3: Summary statistics of patient score improvements following vision therapy treatments for post concussion syndrome grouped by number of VT sessions $(n=56)$.

\begin{tabular}{|c|c|c|c|c|c|c|c|c|}
\hline Number of VT Sessions & \multicolumn{2}{|c|}{$5-20$} & \multicolumn{2}{|c|}{$5-10$} & \multicolumn{2}{|c|}{$12-17$} & \multicolumn{2}{|c|}{20} \\
\hline Count (n) & \multicolumn{2}{|c|}{56} & \multicolumn{2}{|c|}{29} & \multicolumn{2}{|c|}{10} & \multicolumn{2}{|c|}{17} \\
\hline & avg & std & avg & std & avg & std & avg & std \\
\hline Months since injury & 15.1 & 14.2 & 14.3 & 13.2 & 14.9 & 18.6 & 16.4 & 13.7 \\
\hline \multirow[t]{2}{*}{ Duration of program (weeks) } & 15.2 & 4.86 & 11.2 & 2.13 & 16.0 & 1.56 & 21.5 & 0.94 \\
\hline & \multicolumn{8}{|c|}{ Percent Score Improvement } \\
\hline Measure & avg & std & avg & std & avg & std & avg & std \\
\hline $\mathrm{NPC}(\mathrm{cm})$ & $65 \%$ & 20 & $67 \%$ & 18 & $64 \%$ & 25 & $62 \%$ & 21 \\
\hline Vergence Facility (cpm) & $83 \%$ & 27 & $77 \%$ & 32 & $83 \%$ & 23 & $94 \%$ & 12 \\
\hline Accomodative Facility $(\mathrm{cpm}) \delta$ & $71 \%$ & 26 & $66 \%$ & 27 & $85 \%$ & 17 & $72 \%$ & 31 \\
\hline King Devick (s) & $27 \%$ & 16 & $23 \%$ & 16 & $30 \%$ & 12 & $31 \%$ & 19 \\
\hline Stereopsis- Near & $39 \%$ & 23 & $34 \%$ & 22 & $54 \%$ & 27 & $38 \%$ & 20 \\
\hline Stereopsis- Dist & $41 \%$ & 31 & $29 \%$ & 27 & $61 \%$ & 33 & $50 \%$ & 28 \\
\hline \multirow[t]{2}{*}{ CISS (Score) } & $49 \%$ & 14 & $50 \%$ & 16 & $55 \%$ & 14 & $46 \%$ & 9 \\
\hline & \multicolumn{8}{|c|}{ Score Improvement } \\
\hline Measure & avg & std & avg & std & avg & std & avg & std \\
\hline PFV Break D (6m) & 11.4 & 7.42 & 10.8 & 8.08 & 10.0 & 5.25 & 13.1 & 7.36 \\
\hline PFV Recovery - D (6m) & 10.4 & 6.44 & 10.3 & 7.43 & 9.80 & 4.57 & 10.9 & 5.81 \\
\hline NFV Break D $(40 \mathrm{~cm})$ & 4.70 & 3.35 & 4.59 & 3.59 & 4.60 & 3.66 & 4.94 & 2.88 \\
\hline NFV Recovery- D $(40 \mathrm{~cm})$ & 4.88 & 3.03 & 5.10 & 3.45 & 3.60 & 2.84 & 5.24 & 2.25 \\
\hline PFV Break- D (40cm) & 18.2 & 8.92 & 17.2 & 8.56 & 16.6 & 10.8 & 20.6 & 8.42 \\
\hline PFV Recovery- D (40cm)Near & 16.9 & 9.13 & 15.9 & 9.71 & 16.4 & 9.65 & 18.8 & 7.95 \\
\hline NFV Break- D (40cm) & 10.3 & 6.33 & 9.66 & 5.10 & 9.60 & 6.31 & 11.8 & 8.14 \\
\hline NFV Recovery- D $(40 \mathrm{~cm})$ & 10.3 & 6.17 & 9.93 & 5.08 & 8.90 & 7.34 & 11.8 & 7.17 \\
\hline
\end{tabular}

$\delta n=22$, average age of $28.4 \pm 10.1$ 


\section{REFERENCES}

1. Mayo Clinic. Post-concussion syndrome. https://www.mayoclinic. org/diseases-conditions/post-concussion-syndrome/symptomscauses/syc-20353352 (accessed 5 June 2018).

2. Gallaway M, Scheiman M, Mitchell GL. Vision therapy for postconcussion vision disorders. Optom Vis Sci 2017 Jan;94(1):68-73.

3. Greenwald BD, Kapoor N, Singh AD. Visual impairments in the first year after traumatic brain injury. Brain Inj 2012;26(11):1338-59.

4. Skaler T, Niland P. Optometric vision therapy in rehabilitation of cognitive dysfunctions caused by traumatic brain injury. Scottish Intercollegiate Guidelines Network; 2015 https://www.acc.co.nz/ assets/research/f4d5ec9777/optometric-therapy-tbi-review.pdf (accessed 18 October 2019).

5. Broglio SP, Collins MW, Williams RM, Mucha A, Kontos AP. Current and emerging rehabilitation for concussion. a review of the evidence. Clin Sports Med 2015 Apr;34(2):213-31.

6. Lew HL, Pogoda TK, Baker E, et al. Prevalence of dual sensory impairment and its association with traumatic brain injury and blast exposure in OEF/OIF veterans. J Head Trauma Rehabil 2011 Nov-Dec;26(6):489-96.

7. Hartvigsen J, Boyle E, Cassidy JD, Carroll LJ. Mild traumatic brain injury after motor vehicle collisions: what are the symptoms and who treats them? A population-based 1-year inception cohort study. Arch Phys Med Rehabil 2014 Mar;95(3 Suppl):S286-94.

8. Dougherty AL, MacGregor AJ, Han PP, Heltemes KJ, Galarneau MR. Visual dysfunction following blast-related traumatic brain injury from the battlefield. Brain Inj 2011;25(1):8-13.

9. Bulson R, Jun W, Hayes J. Visual symptomatology and referral patterns for Operation Iraqi Freedom and Operation Enduring Freedom veterans with traumatic brain injury. $J$ Rehabil Res Dev 2012;49(7):1075-82.

10. Ciuffreda KJ, Kapoor N, Rutner D, Suchoff IB, Han ME, Craig S. Occurrence of oculomotor dysfunctions in acquired brain injury: a retrospective analysis. Optometry 2007 Apr;78(4):155-61.

11. Master CL, Scheiman M, Gallaway M, et al. Vision diagnoses are common after concussion in adolescents. Clin Pediatr (Phila) 2016 Mar;55(3):260-7.

12. Porcar E, Martinez-Palomera A. Prevalence of general binocular dysfunctions in a population of university students. Optom Vis Sci 1997 Feb;74(2):111-3.

13. Scheiman M, Gwiazda J, Li T. Non-surgical interventions for convergence insufficiency. Cochrane Database Syst Rev 2011 Mar 16;(3):CD006768.

14. Lee SH, Moon BY, Cho HG. Improvement of vergence movements by vision therapy decreases K-ARS scores of symptomatic ADHD children. J Phys Ther Sci 2014 Feb;26(2):223-7.
15. Scheiman M, Wick B, eds. Clinical management of binocular vision: Heterophoric, accommodative, and eye movement disorders. Philadelphia: Lippincott, Williams \& Wilkins, 2008.

16. Ciuffreda KJ, Rutner D, Kapoor N, Suchoff IB, Craig S, Han ME. Vision therapy for oculomotor dysfunctions in acquired brain injury: a retrospective analysis. Optometry 2008 Jan;79(1):18-22.

17. Thiagarajan P, Ciuffreda KJ. Effect of oculomotor rehabilitation on vergence responsivity in mild traumatic brain injury. $J$ Rehabil Res Dev 2013;50(9):1223-40.

18. Thiagarajan P, Ciuffreda KJ. Versional eye tracking in mild traumatic brain injury (mTBI): Effects of oculomotor training (OMT). Brain Inj 2014;28(7):930-43.

19. Thiagarajan P, Ciuffreda KJ. Effect of oculomotor rehabilitation on accommodative responsivity in mild traumatic brain injury. $J$ Rehabil Res Dev 2014;51(2):175-91.

20. Thiagarajan P, Ciuffreda KJ, Capo-Aponte JE, Ludlam DP, Kapoor N. Oculomotor neurorehabilitation for reading in mild traumatic brain injury (mTBI): An integrative approach. NeuroRehabilitation 2014;34(1):129-46.

21. Alvarez TL, Jaswal R, Gohel S, Biswal BB. Functional activity within the frontal eye fields, posterior parietal cortex, and cerebellar vermis significantly correlates to symmetrical vergence peak velocity: An ROI-based, fMRI study of vergence training. Front Integr Neurosci 2014 Jun 17;8:50.

22. Lacroix Z, Leat SJ, Christian LW. Role of primary care optometrists in the assessment and management of patients with traumatic brain injuries in Canada. Can J Optom 2016;80(1):13-7.

23. Elliott BD, ed. Clinical procedures in primary eye care, 3 rd edn. Philadelphia, Elsevier Butterworth-Heinemann, 2007.

24. Convergence Insufficiency Treatment Trial Study Group. Randomized clinical trial of treatments for symptomatic convergence insufficiency in children. Arch Ophthalmol 2008 Oct;126(10):1336-49.

25. Press LJ. Applied concepts in vision therapy. Optometric Extension Program Fndtn; OEP Edition, 1997.

26. Rosner B, Glynn RJ, Lee ML. The Wilcoxon signed rank test for paired comparisons of clustered data. Biometrics 2006 Mar;62(1):185-92.

27. Horwood AM, Toor SS, Riddell PM. Change in convergence and accommodation after two weeks of eye exercises in typical young adults. J AAPOS 2014 Apr;18(2):162-8. 